\title{
A Nuclear Defect in the 4p16 Region Predisposes to Multiple Mitochondrial DNA Deletions in Families with Wolfram Syndrome
}

\author{
Antoni Barrientos, ${ }^{\star \ddagger}$ Víctor Volpini,, Jordi Casademont, ${ }^{*}$ David Genís, ${ }^{\S}$ Josep-Maria Manzanares, ${ }^{\mathbb{} I s i d r e ~ F e r r e r, ~} \|$ Jordi Corral, ${ }^{\ddagger}$ \\ Francesc Cardellach, ${ }^{*}$ Alvaro Urbano-Márquez, ${ }^{*}$ Xavier Estivill,, ${ }^{\ddagger \star}$ and Virginia Nunes ${ }^{\ddagger}$ \\ *Grup d'Investigació Muscular, Departament de Medicina, Hospital Clínic i Provincial i Universitat de Barcelona, Villarroel 170, 08036 \\ Barcelona, Spain; ${ }^{\ddagger}$ Departament de Genètica Molecular, Institut de Recerca Oncologica (IRO), 08907 Hospitalet del Llobregat, Barcelona, \\ Spain; ${ }^{\S}$ Unitat de Neurologia, Hospital Josep Trueta, 17007 Girona, Spain; "Unitat de Neuropatologia, Servei d'Anatomia Patologica, \\ Hospital Princeps d'Espanya, 08907 Hospitalet del Llobregat, Barcelona, Spain; and Serveis de ${ }^{\mathbb{I}}$ Endocrinologia and **Genètica, Hospital \\ Clínic i Provincial de Barcelona, Villarroel 170, 08036 Barcelona, Spain
}

\begin{abstract}
Wolfram syndrome is a progressive neurodegenerative disorder transmitted in an autosomal recessive mode. We report two Wolfram syndrome families harboring multiple deletions of mitochondrial DNA. The deletions reached percentages as high as $\mathbf{8 5 - 9 0 \%}$ in affected tissues such as the central nervous system of one patient, while in other tissues from the same patient and from other members of the family, the percentages of deleted mitochondrial DNA genomes were only $1-10 \%$. Recently, a Wolfram syndrome gene has been linked to markers on $4 \mathrm{p} 16$. In both families linkage between the disease locus and $4 \mathrm{p} 16$ markers gave a maximum multipoint lod score of 3.79 at $\theta=0(P<0.03)$ with respect to D4S431. In these families, the syndrome was caused by mutations in this nucleus-encoded gene which deleteriously interacts with the mitochondrial genome. This is the first evidence of the implication of both genomes in a recessive disease. (J. Clin. Invest. 1996. 97:1570-1576.) Key words: neurodegenerative disease $\cdot$ OXPHOS • autosomal recessive inheritance $\cdot$ linkage $\cdot$ DIDMOAD
\end{abstract}

\section{Introduction}

Wolfram syndrome (WS) ${ }^{1}$ (MIM 222300) (1) was described in 1938 by Wolfram and Wagener (2). The most frequent manifestations of the syndrome are diabetes insipidus, insulindependent diabetes mellitus (IDDM), bilateral progressive optic atrophy, and deafness. Nonetheless, such manifestations are not always present, yet the association with other diverse neurological and psychiatric features, including ataxia, peripheral neuropathy, urinary tract atony, dementia, depression,

Antoni Barrientos and Víctor Volpini have contributed equally to this study.

Address correspondence to Virginia Nunes, Departament de Genètica Molecular, Institut de Recerca Oncològica (IRO), Autovia de Castelldefels, Km 2.7, 08907 Hospitalet del Llobregat, Barcelona, Spain. Phone: 34-3-3357152; FAX: 34-3-2632251.

Received for publication 25 August 1995 and accepted in revised form 8 January 1996.

1. Abbreviations used in this paper: IDDM, insulin-dependent diabetes mellitus; mtDNA, mitochondrial DNA; WS, Wolfram syndrome.

J. Clin. Invest.

(c) The American Society for Clinical Investigation, Inc. 0021-9738/96/03/1570/07 \$2.00

Volume 97, Number 7, April 1, 1996, 1570-1576 psychosis, and aggressive behavior, is frequent (3-6). Diagnosis is usually established in one or several siblings from unaffected parents with some degree of consanguinity, suggesting an autosomal recessive mode of transmission $(6,7)$. Recently, a Wolfram syndrome gene has been linked to markers on the short arm of human chromosome 4 (reference 8 ). Nevertheless, the wide spectrum of clinical expression, affecting several organs and tissues, suggests mitochondrial DNA (mtDNA) involvement, a fact that has already been proven in sporadic cases $(9,10)$.

Human mtDNA is maternally transmitted (11) and contains 13 structural genes encoding for subunits of the respiratory chain enzyme complexes. The majority of the respiratory chain enzyme subunits and all the proteins required for the replication, transcription, and translation of mtDNA are encoded in the nucleus. The function of the respiratory chain is thus regulated by both mitochondrial and nuclear genes. A nuclear gene defect that interferes with mtDNA integrity has been suggested in several families with autosomal dominant (12) or recessive $(13,14)$ inheritance associated with multiple mtDNA deletions. Recently, the first autosomal dominant locus that predisposes to mtDNA deletions has been localized to chromosome 10q in families with progressive external ophthalmoplegia (15).

We report two WS families harboring multiple deletions of mtDNA in which the disease locus is linked to 4p16. The findings in these families demonstrate that an autosomal locus, localized to $4 \mathrm{p} 16$, predisposes to mtDNA deletions resulting in a semidominant model of inheritance causing WS when both homologous chromosomes are affected in one individual. This is the first direct evidence of the involvement of mitochondrial and nuclear genomes in an autosomal recessive disorder.

Case report. Two kindreds of Spanish Caucasian origin, WS1 and WS2 (Fig. 1), were studied.

In family WS1, Wolfram syndrome was diagnosed in four sisters whose parents were first cousins. All the sisters first presented with IDDM and dyschromatopsia followed by severe optic atrophy in their thirties. Later on they developed psychiatric abnormalities in the form of anxiety, abnormal behavior, anterograde amnesia, sphincter disturbances, anosmia, walking instability, tremor, dysphagia, and swallowing difficulties. Sisters IV-6 and IV-7 died after food aspiration and IV-4 suffered eight aspirative pneumonias. The autopsy study of IV-6 disclosed severe brain and posterior fossa structure atrophy. Olfactory, optic, and cochlear nerves appeared demyelinated and atrophic. Neuron loss was observed in preoptic, paraventricular, and thalamic nuclei, dorsal nucleus of the vagus, ambiguous nucleus, and olivary and pontine nuclei. There was Purkinje cell loss in cerebellum and reduced motor neuron 


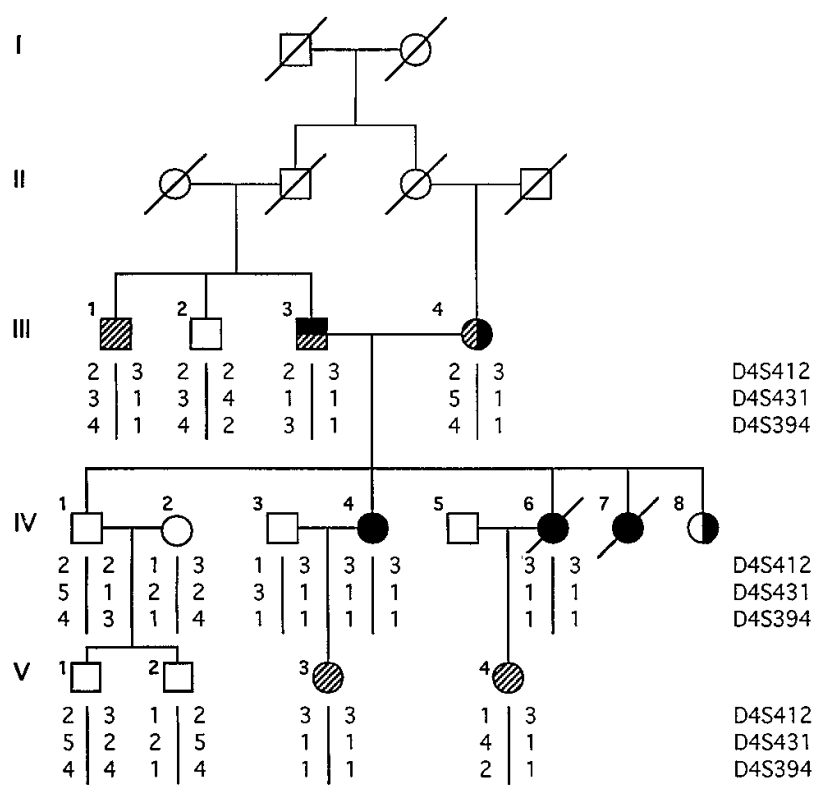

number in spinal cord. The periventricular white matter was demyelinated.

The patient in the nonconsanguineous WS2 family was a 27 -yr-old male. At 8 yr of age, he developed IDDM. When he was 16 , bilateral atrophy of optic nerves and neurosensorial deafness for high frequencies were detected. 2 yr later, the visual loss was almost complete and he developed diabetes insipidus when he was $23 \mathrm{yr}$ old.

\section{Methods}

Biochemical studies. Lymphocytes from all members of family WS2 were isolated from $10 \mathrm{ml}$ blood on a Ficoll cushion. The polarographic studies were performed at $30^{\circ} \mathrm{C}$ as previously described (16). Assays of intact cell respiration and mitochondrial substrate (glutamate, pyruvate-malate, succinate, and glycerol-3-phosphate) oxidation by detergent-permeabilized cells were performed. Enzymatic assays of NADH cytochrome $c$ reductase, cytochrome $c$ oxidase, and succinate cytochrome $c$ reductase activities were carried out spectrophotometrically (UVIKON 860; Kontron AS, Zurich, Switzerland) as described elsewhere (16). 11 healthy individuals with ages ranging from 17 to $49 \mathrm{yr}$ were taken as controls.

$m t D N A$ studies. Total DNA was extracted from blood samples of members of both families and from skeletal muscle, liver, and several areas of the brain from the autopsied WS1 patient IV6. mtDNA was linearized by digestion with PvuII restriction endonuclease (cut in nucleotide [nt] 2650) (Boehringer Mannheim Corp., Indianapolis, IN), separated through $0.8 \%$ agarose gel electrophoresis, blotted onto nylon membrane (Biodyne A; Pall Biosupport Corp., Glen Cove, NY), hybridized with whole human mtDNA labeled with $\left[{ }^{32} \mathrm{P}\right] \alpha \mathrm{dCTP}$ (Amersham Corp., Arlington Heights, IL), and autoradiographed. The filter was subsequently hybridized with an mtDNA probe spanning from nucleotide 459 to 3334, and then with an mtDNA probe spanning from nucleotide 10122 to 11587 (PR2). mtDNA was also linearized by digestion with SnaBI restriction endonuclease (cut in $\mathrm{nt}$ 10736) (Boehringer Mannheim Corp.) (Fig. 2) and subsequently hybridized with probes PR1 (nt 4214-5791) and PR2 (Fig. 2). Several pairs of primers (e.g., L820 [nt 8200-8220] and H1619 [nt 16210-

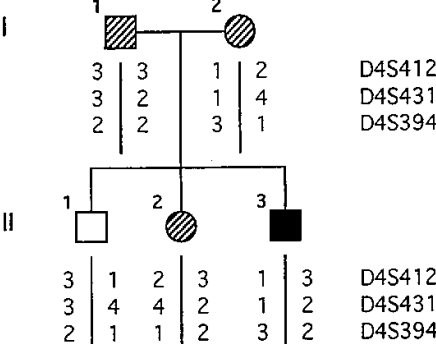

Figure 1. Pedigree of families identification number is shown above and left of the symbol. Dashed symbols represent clinically asymptomatic patients with mtDNA deletions, filled symbols represent clinically affected patients with mtDNA deletions, and open symbols represent normal individuals without mtDNA deletions. Vertically half-filled symbols identify patients whose only clinical problem was diabetes mellitus, and horizontally half-filled symbols identify patients with neurosensory hearing loss. Numbers under symbols represent the haplotypes for markers D4S412, D4S431, and D4S394.
16190], PM1 [8335-8355], and PM2 [14110-13990]) (Fig. 2) were used to PCR amplify regions of mtDNA as previously described $(13,17)$. The fragments containing the deletion break points were run on 5-8\% polyacrylamide gels. The most represented bands were cut out from the gel, reamplified with the same primers, and purified using Qiaquick Spin PCR purification Kit (QIAGEN Inc., Chatsworth, CA). Direct automatic sequencing was performed with 3.5 pmol of sequencing primers using a Taq Dye Deoxy ${ }^{\mathrm{TM}}$ Terminator Sequencing kit (No. 401113; Applied Biosystems, Foster City, CA) according to manufacturer's recommendations. Sequencing primers were those used in the original PCR amplifications. Data analyses were performed with the 373A sequencing software from ABI Advanced Biotechnologies, Inc. (Columbia, MD). To quantify the relative proportion of normal and deleted mtDNA, Southern autoradiographs were scanned, correcting for the background, with a Préférence/DVS-1500 densitometer (Sebia, Paris, France). When the deletions were not detected by Southern blot, the relative proportion of deleted versus total mtDNA was estimated using a semiquantitative PCR-based method modified after DiDonato et al. (18). The number of amplification cycles was chosen to take into account the PCR exponential phase. Total DNA was diluted to $1 \mu \mathrm{g} / \mu \mathrm{l}$ in $1 \times 10 \mathrm{mM}$ TRIS-HCl/1 mM EDTA (pH 8.0). Serial 10-fold dilutions were made down to 100 $\mathrm{pg} / \mu \mathrm{l}$. For each dilution, a differential PCR amplification was obtained using two pairs of primers. The primers PR2 $2_{1}$ (nt 10122-10143) and $\mathrm{H}_{\mathrm{Arg}}$ (nt 10522-10504) were used to amplify a 382-bp fragment, internal to the deletions sequenced in patients; the second pair of primers ( $\mathrm{L}_{\mathrm{Leu}-1}$-nt 3164-3183- and $\mathrm{H}_{\mathrm{Leu}-1}$-nt 3353-3334) were used to amplify a 170-bp fragment external to the deletion. The 170-bp fragment corresponds to the amplification product generated from the total mtDNA template (TT) contained in each sample. The 382-bp fragment was considered as the nondeleted mtDNA (NDT). $8 \mu \mathrm{l}$ of the PCR amplifications was run in a $0.8 \%$ agarose gel and ethidium bromide stained, and the fragments were visualized under ultraviolet light. Densitometric analyses were performed with a scanner (ScanJet IIcx; Hewlett-Packard Co., Palo Alto, CA) and with a densitometer (Préférence/DVS 1500; Sebia). After correction for the length of the fragments, the series of densitometrical values corresponding to TT and NDT amplifications were plotted as a function of the logarithm of template dilutions and a linear regression analysis was performed 

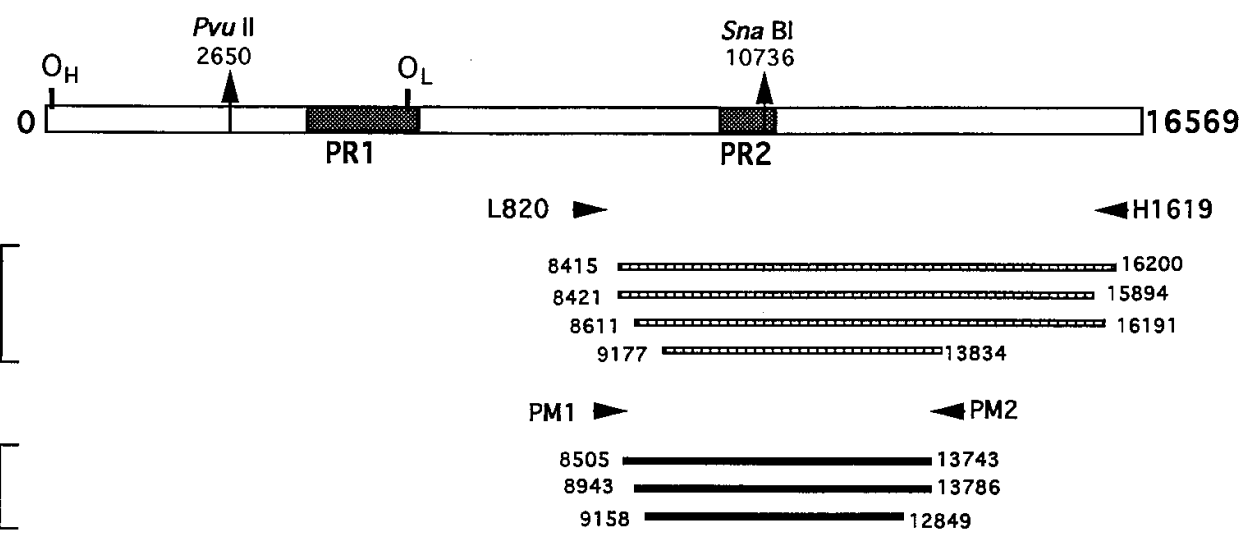

Figure 2. Schematic representation of the mitochondrial genome. The probes PR1 and PR2 and the restriction sites of the enzymes PvuII and SnaBI are placed. At the bottom, the arrows represent some of the primer pair used in the PCR analyses and the location of the sequenced deletions for the families: $a$, WS1; $b$, WS2.

for the two series. The coefficient of linear correlation was, in all cases, consistently $>0.97$. The percentage of the deleted mtDNA versus total mtDNA was obtained as described elsewhere (18).

Genotyping studies. To analyze microsatellites D4S412, D4S431, and D4S394, the dinucleotide repeat alleles were PCR-amplified with primers obtained from the Human Genome Research Center (Evry, France) in a final reaction volume of $25 \mu \mathrm{l}$. The reaction contained $100 \mathrm{ng}$ of DNA, 1 pmol of the $\left[{ }^{33} \mathrm{P}\right] \gamma \mathrm{dATP}$ end labeled forward primer, $10 \mathrm{pmol}$ of the reverse primer, $0.5 \mathrm{U}$ of Taq polymerase (Boehringer Mannheim Corp.), $200 \mu \mathrm{M}$ of each of the four deoxynucleotides, and Taq polymerase incubation buffer (Boehringer Mannheim Corp.). Samples were amplified in a thermocycler (model 9600; Perkin-Elmer Corp., Norwalk, CT) for 35 cycles of $25 \mathrm{~s}$ at $95^{\circ} \mathrm{C}, 25 \mathrm{~s}$ at $56^{\circ} \mathrm{C}$, and $25 \mathrm{~s}$ at $74^{\circ} \mathrm{C}$, followed by a 5 -min final extension at $74^{\circ} \mathrm{C}$. $16 \mu$ l of dye containing $95 \%$ formamide was added to the reaction, the samples were then denatured at $95^{\circ} \mathrm{C}$ for $1-2 \mathrm{~min}$ and ice-cooled immediately. Amplification products were then electrophoresed in $6 \%$ denaturing polyacrylamide gels at $1,500 \mathrm{~V}$. Gels were transferred, vacuum dried, and exposed to radiographic film. Allele sizes were determined from a CEPH control individual of known genotype (1347-02).

Linkage studies. We performed linkage studies using the lod score method (19). To facilitate the calculation, we used the programs MLINK, ILINK, and LINKMAP from FASTLINK SOFTWARE V2.2 (20). We used three 4p16 markers (D4S412, D4S431, and D4S394) which define a region from which linkage to WS locus has been demonstrated previously (8). The deleterious allele frequency was set at 0.01 and all marker alleles were considered to be equally frequent. Varying the allele frequencies did not significantly alter the lod scores, due to the small number of untyped individuals in the pedigrees. It was necessary, due to computational constraints, to downcode alleles to a maximum of three for $D 4 S 412$, five for $D 4 S 431$, and four for D4S394. In the multipoint analysis a fixed distance of $6 \mathrm{cM}$ between each of the three loci was considered in accordance with the reported data (21). We used computer simulation methods to calculate the signification ( $P$ value) associated with the multipoint lod score $(22,23)$. All the programs were run in a UNIX SPARC SOLARIS 2.4 computer.

\section{Results}

Enzymological studies. The oxidative phosphorylation pathway in lymphocytes from the WS patient of family WS2 was studied. When pyruvate, glutamate, or succinate was used as substrate for mitochondrial oxidation in detergent-permeabilized cells, a significant functional deficiency was demonstrated $(36,45$, and $24 \%$, respectively). The ratios between the different substrates were normal. Enzymatic assays showed a decrease of $22 \%$ for NADH cytochrome $c$ reductase, $29 \%$ for cytochrome $c$ oxidase, and $9 \%$ for succinate cytochrome $c$ reductase activities. His parents, brother, and sister showed normal mitochondrial function.

mtDNA studies in family WS1. Southern blot analysis of mtDNA probed with the whole mtDNA demonstrated multiple deletions in all the areas of the central nervous system (CNS) analyzed from the autopsy study of patient IV-6. The deletions ranged from 1.5 to $10 \mathrm{~kb}$ (Fig. $3 a$ ). When the filter was hybridized with PR2, a probe internal to the deletions, no abnormal bands were found (Fig. $3 b$ ). Liver and muscle from IV-6 patient as well as lymphocytes from the remaining members of the family examined did not reveal any abnormal band. To demonstrate the nature of the mtDNA rearrangements found in the brain of the patient, we digested mtDNA with SnaBI, an enzyme that cuts at nt 10736, internal to the rearrangements. When we hybridized with PR1, a probe external to the deletions, two bands were found: one of $16.5 \mathrm{~kb}$ corresponding to the wild-type band and some comigrating undigested rearranged molecules, and another of $10 \mathrm{~kb}$ corresponding to the other undigested rearranged molecules (Fig. 3 c). A normal pattern after hybridization with PR2 indicates that rearrangements were deletions and not duplications. The
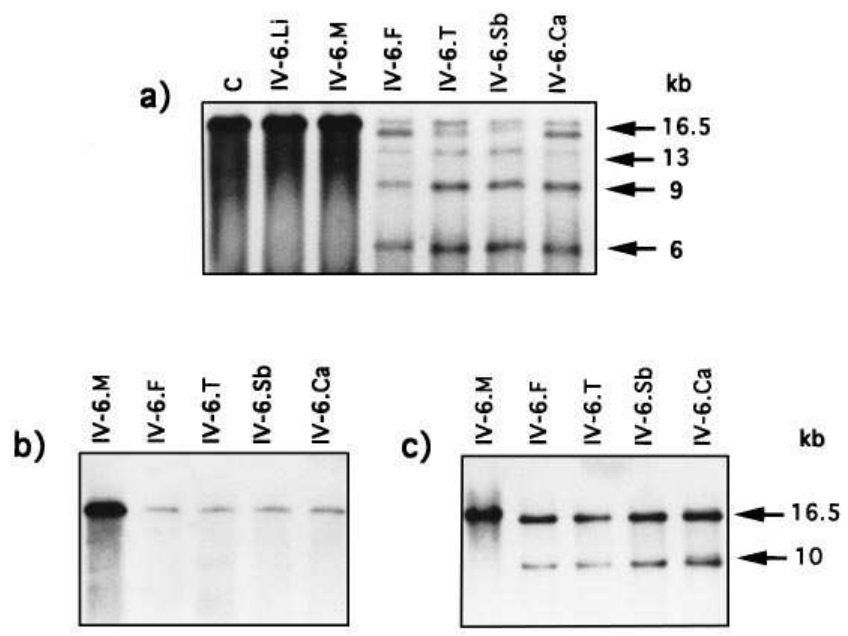

Figure 3. Southern blot analysis of tissues obtained from patient IV-6 of family WS1. (a) PvuII digestion probed with whole mtDNA; (b) SnaBI digestion hybridized with a probe spanning from nt 10122 to 11587 (PR2); (c) SnaBI digestion hybridized with a probe spanning from nt 4214 to 5791 (PR1). C, control; $L i$, liver; $M$, skeletal muscle; $F$, frontal lobe; $T$, temporal lobe; $S b$, white matter; $C a$, caudate. 
A

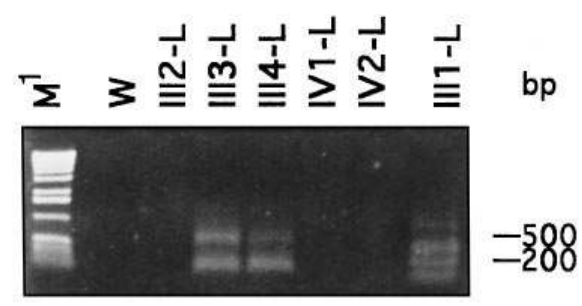

B
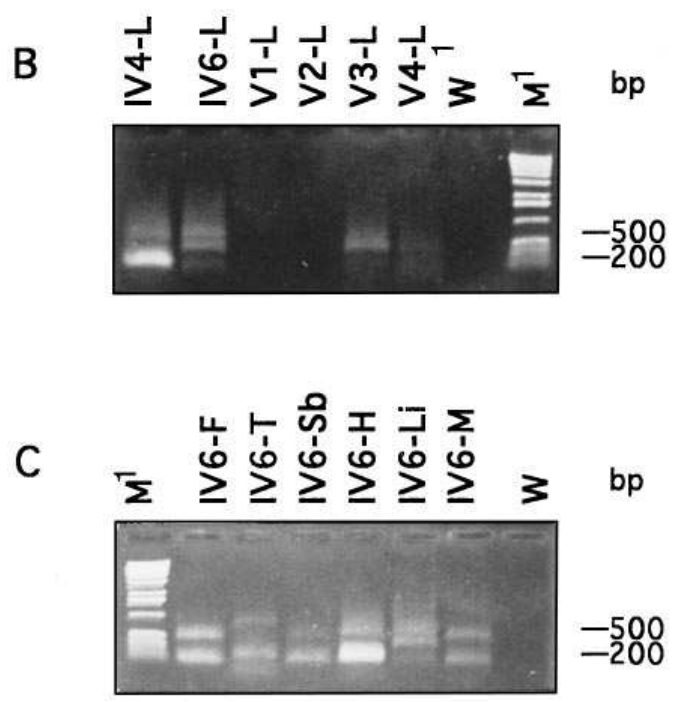

Figure 4. PCR analyses of mtDNA from family WS1 with primers L820 (nt 8200-8220) and H1619 (nt 16210-16190). ( $A$ and $B$ ) Analyses of all the members of the family; $(C)$ analyses of different tissues from patient IV-6. PCR analyses revealed several fragments ranging from 200 to $500 \mathrm{bp}$ approximately, corresponding to multiple deletions of 7.5-7.8 kb. The numbers are the same as those in the pedigree of family WS1 (Fig. 1). $L$, lymphocytes; $L i$, liver; $M^{1}$, marker; $M$, skeletal muscle; $F$, frontal lobe; $H$, hippocampus; $T$, temporal lobe; $S b$, white matter; $W$, control without DNA.

fact that some undigested mtDNA molecules appear to have the same size in the SnaBI digestion is probably due to the secondary structure adopted by the mtDNA molecules with different deletions allowing them to comigrate. PCR analyses with primers distributed along the mtDNA genome disclosed the presence of several fragments confirming the existence of multiple deletions. For example, PCR analyses with primers

Table I. Percentage of Deleted mtDNA in Different Tissues from Patient IV-6 of the WSI Family

\begin{tabular}{lc}
\hline \multicolumn{1}{c}{ Tissue } & Percentage of deleted mtDNA \\
\hline Liver & $\sim 5$ \\
Skeletal muscle & $\sim 5$ \\
Lymphocytes & $\sim 5$ \\
Brain areas & \\
$\quad$ Frontal & 90 \\
Caudate & 85 \\
White matter & 87 \\
Temporal & 86 \\
Putamen & 91 \\
& \\
\hline
\end{tabular}

L820 and H1619 revealed several fragments ranging from 200 to $500 \mathrm{bp}$ approximately, corresponding to multiple deletions of 7.5-7.8 kb (Fig. 4). These fragments were found in lymphocytes of the family members with dashed symbols in the pedigree (Fig. 1) and in all tissues studied from patient IV-6. The results were reproduced with nested PCRs. The deletions found by PCR were similar in size, but not identical, in the different members of the family and different tissues of patient IV-6. The proportion of deleted molecules ranged between 85 and $90 \%$ in affected tissues such as the CNS from patient IV-6 (Table I), while in other tissues (liver and skeletal muscle) from the same patient, as well as lymphocytes from other members of the family, the proportion ranged from 1 to $10 \%$. Several bands detected by Southern blot and PCR analysis in brain of patient IV-6 were reamplified and sequenced. Deletions were flanked by perfect short direct repeats of 5-9 bp (Figs. 2 and 5).

mtDNA studies in family WS2. PvuII Southern blot analysis

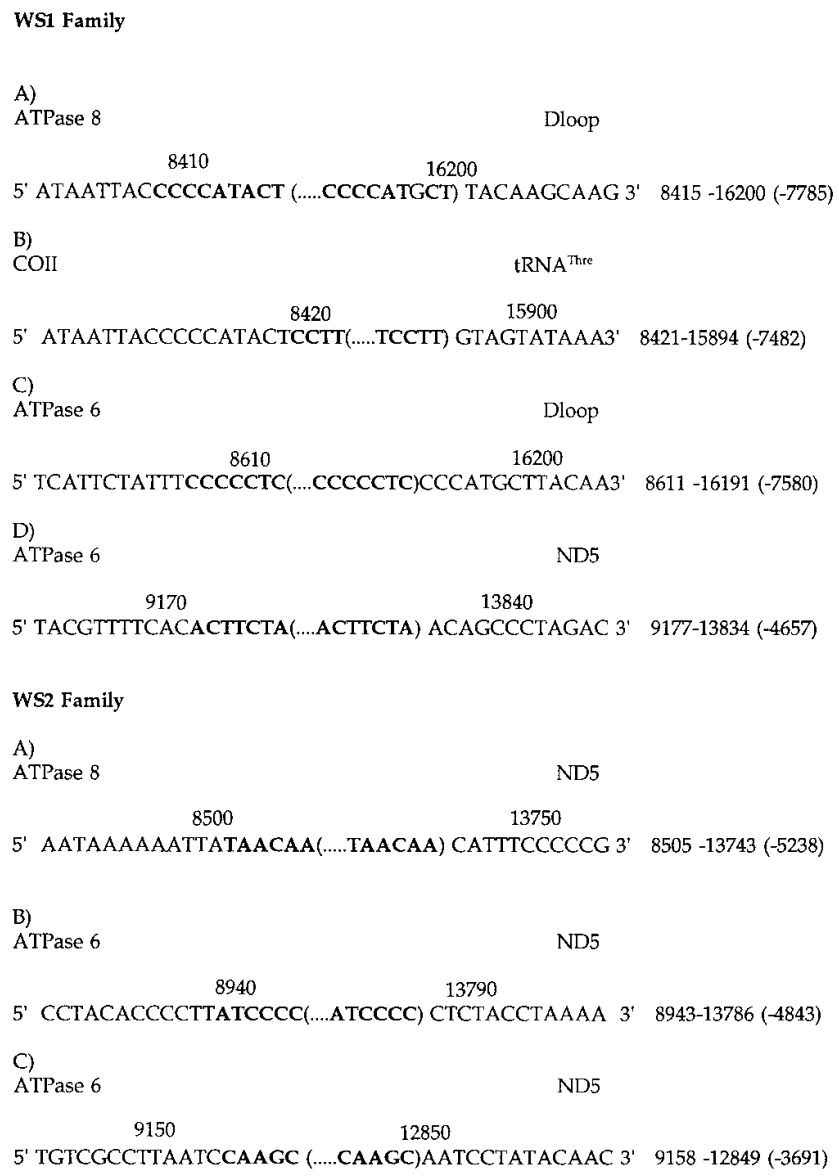

Figure 5. Sequence of the mtDNA deletions found in families WS1 and WS2. Nucleotide numbering is in accordance with the Cambridge human mtDNA sequence of the $\mathrm{L}$ strand, reading $5^{\prime}$ to $3^{\prime}$ (reference 31). Deleted fragment is indicated in parentheses. Direct repeats are bold. On the right hand side the nucleotides $5^{\prime}$ and $3^{\prime}$ limiting the deletions (the length of the deletion is in parentheses) are shown. The genes involved in the break point of any deletion are shown above the $5^{\prime}$ and $3^{\prime}$ ends of the sequences. ND5, NADH dehydrogenase subunit 5 gene; ATPase 6, ATPase subunit 6 gene; ATPase 8 , ATPase subunit 8 gene; Dloop, deplacement loop noncoding region; $C O I I$, cytochrome $c$ oxidase subunit II gene; $t R N A^{\text {Thre }}$, threonine tRNA gene. 
of mtDNA from lymphocytes hybridized with the whole mtDNA probe disclosed abnormal bands of 7-14 kb corresponding to multiple deletions of $2.5-9.5 \mathrm{~kb}$ in the patient (II-3) and his sister (II-2) (Fig. 6) accounting for 55 and $16 \%$ of whole mtDNA molecules, respectively. Hybridization with PR2 disclosed a normal pattern in all members. PCR analyses with primers distributed along the mtDNA genome disclosed the presence of several fragments confirming the existence of multiple deletions. For example, PCR analysis using primers PM1 and PM2 showed in all individuals, except II-1, several fragments ranging from 400 to $1,800 \mathrm{bp}$ approximately, representing multiple deletions from 3.8 to $5.2 \mathrm{~kb}$ that were similar in size, but not identical, in the different members of the family (Fig. 7). The results were confirmed with nested PCRs. Several bands detected by Southern blot and PCR analysis in lymphocytes of patient II-3 were reamplified and sequenced. Deletions were flanked by perfect short direct repeats of 5-6 bp (Figs. 2 and 5).

Linkage between Wolfram syndrome and markers on $4 p 16$. Wolfram syndrome is considered to be transmitted in an autosomal recessive way. In accordance with the presence of mtDNA rearrangements and disease signs and symptoms, we hypothesized a model with three different phenotypes which could be explained by a nuclear biallelic gene (+/D) in an autosomal semidominant form of transmission. Genotype $+/ \mathrm{D}$ would produce mtDNA deletions but no disease manifestations, D/D would produce mtDNA deletions and disease manifestations, and $+/+$ would produce no deletions and a healthy individual (wild-type). This mode of inheritance preserves the classical autosomal recessive model of transmission for the disease and allows the inclusion of mtDNA deletions as a part of Wolfram syndrome in our families. We performed linkage analysis using three 4p16 markers (D4S412, D4S431, and D4S394) with $6 \mathrm{cM}$ of genetic distance between them (8). In the two-point analyses (disease locus versus loci markers) the highest lod score corresponded to $D 4 S 394$ with $\mathrm{Z}_{\max }=2.67$ at $\theta=0$. We found linkage between the disease locus and the analyzed 4p16 markers with a maximum multipoint lod score of 3.79 at $\theta=0$ with respect to marker D4S431 $(P<0.03)$. This demonstrated linkage between the WS trait locus in these families and the region defined by the markers used in the multipoint analysis.

\section{Discussion}

This study demonstrates the presence of familial multiple mtDNA deletions in two pedigrees with autosomal recessive

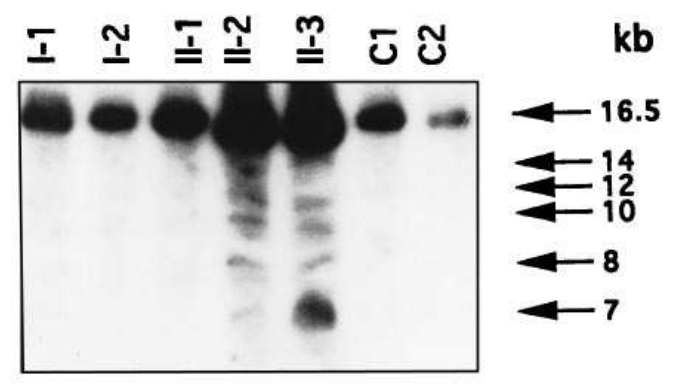

Figure 6. PvuII Southern blot analysis of lymphocytes from family WS2 probed with whole mtDNA. The numbers are the same as those in the pedigree of family WS2 (Fig. 1). C1 and C2 are controls.
Figure 7. PCR analyses of mtDNA from lymphocytes from members of family WS2 with primers PM1 (nt

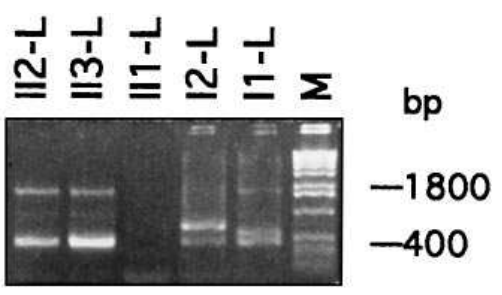
8335-8355) and PM2 (nt 14010-13990). The numbers are the same as those in the pedigree of family WS2 (Fig. 1). $L$, lymphocytes; $M$, marker.

Wolfram syndrome as well as linkage to the $4 \mathrm{p} 16$ region as defined by markers D4S431 and D4S394. Our results are direct evidence of the implication of both nuclear and mitochondrial genomes, such that Wolfram syndrome is the first autosomal recessive disorder in which a dual involvement is demonstrated.

Linkage of the gene for WS to the 4p16 region had been shown previously (8), but mtDNA analysis in the linked families was not performed or reported. The usual finding in familial disorders associated with mtDNA rearrangements is the presence of multiple deletions in skeletal muscle $(12,14,24)$ or lymphocytes $(24,25)$. In the WS families reported here, the mtDNA deletions appeared in different tissues and proportions. In family WS1, the deletions were present in lymphocytes at a percentage ranging from 1 to $10 \%$. In patient IV-6, the only patient in whom other tissues could be studied, the percentage of deleted molecules in muscle and liver was similar to that found in lymphocytes, but in CNS, the tissue predominantly affected from a clinical point of view, the deleted molecules reached a proportion of $85-90 \%$ (Table I). Respiratory chain diseases considered to be due to mtDNA rearrangements usually present a proportion of deleted molecules of $>50 \%$. Thus, the figure of $85-90 \%$ found in the CNS of patient IV-6 has to be considered the cause, at least in part, of the expression of WS. In family WS2 a high proportion of deleted molecules $(55 \%)$ was found in the lymphocytes of the patient and, in a lower proportion, in their asymptomatic relatives. The relevance of such deletions was further supported by the demonstration of a generalized deficiency of respiratory enzyme activities in patient II-3.

A percentage $<1 \%$ of mtDNA-deleted molecules has been found in normal individuals with increasing age, basically in tissues such as muscle, heart, and CNS $(26,27)$ and has been suggested to be an epiphenomenon related to lifetime oxidative damage. In our families, percentages ranging from 1 to $10 \%$ of deleted mtDNA molecules in both old and young members of the pedigrees and their tissue distribution suggest that factors other than age have to be considered in the origin of multiple mtDNA deletions. These rearrangements, if present in appropriate proportions in target tissues, have to be considered not just as a phenotypic WS marker with no clinical relevance but as an important factor in modulating the expression of the disease.

A disruption in communication between nuclear and mitochondrial genomes had already been demonstrated in autosomal dominant disorders (12) and proposed in cases of autosomal recessive transmission $(13,14)$, but until now this has not been proven. The presence of mtDNA deletions in both paternal and maternal lineages excludes maternal transmission and 
also excludes it being a primary mtDNA disorder. On the contrary, it indicates the presence of a nuclear-encoded factor predisposing to multiple mtDNA deletions transmitted as a Mendelian trait. The expression and penetrance of the WS gene could also be due to its pleiotropic effects or to epigenic factors, either mitochondrial or nuclear in origin.

We propose a model of inheritance for WS based on a semidominant mode of transmission with the presence of mtDNA deletions in heterozygous individuals at a lower proportion than in homozygotes, this low proportion probably not contributing to the clinical phenotype. The incomplete phenotype present in many heterozygous individuals such as deafness and diabetes mellitus (III-1 and III-2 in family WS1) and psychiatric illness (28) would be due to some degree of penetrance of a single copy of the deleterious gene. We found a maximum multipoint lod score of $\mathrm{Z}=3.79$ at $\theta=0$ from D4S431 locus. The significance value is $P<0.03$, which demonstrates linkage between the trait locus and the region defined by the loci markers used in the analysis. As it is not possible to directly infer the associated significance value for lod scores in a multipoint analysis, we obtained it through simulation studies $(22,23)$. The trait locus includes the mitochondrial rearrangements as part of the disease phenotype, and our linkage results demonstrate that this inclusion is not arbitrary, concluding that some WS families show mitochondrial involvement as part of their syndromic manifestations. This conclusion invites discussion on important physiopathological aspects in order to totally explain the phenotype of the syndrome and places the attention on the role of the mitochondria in neurodegenerative disorders. On the other hand, the incidence of the disease has been estimated at 1 out of 10,000 live-born individuals (8). This accounts for a carrier frequency of $H=1 / 100$ according to the Hardy-Weinberg equilibrium. The high frequency of this allele in the general population may account for some cases of common disorders such as diabetes mellitus, deafness, or psychiatric disturbances.

The findings reported in these two WS families point out the implication of a gene related to mitochondrial biogenesis. The three housekeeping genes involved in this process hitherto described $(a)$ the gene encoding for the RNA component of mitochondrial RNA processing protein (29); (b) the mitochondrial transcription factor A; and $(c)$ the mitochondrial single-strand DNA-binding protein (30) have been mapped to chromosomes other than 4 and thus can be excluded as candidate genes. Genes encoding factors implicated in mtDNA turnover or in oxidative metabolism regulation could also be considered as possible candidate genes. How the 4p16 WS locus predisposes to mtDNA rearrangements is still an open question.

\section{Acknowledgments}

The authors thank A. Rötig, P. Rustin, and T. Bourgeron for useful discussion and Helena Kruyer for help in preparing the manuscript.

This work was supported by CICYT SAF 913/93 and 191/95 (to J. Casademont), FIS 94/1563 (to V. Nunes), and DGCYT/PB 93-0019 (to F. Cardellach). Antoni Barrientos is depositary of a grant from Ministerio de Educación y Ciencia PF92 37289410.

\section{References}

1. McKusick, V. 1992. Mendelian Inheritance in Man. Johns Hopkins University Press, Baltimore, MD.
2. Wolfram, D.J., and H.P. Wagener. 1938. Diabetes mellitus and simple optic atrophy among siblings: report of four cases. Mayo Clin. Proc. 1:715-718.

3. Borgna-Pignatti, C., P. Marradi, L. Pinelli, N. Monetti, and C. Patrini. 1989. Thiamine-responsive anaemia in DIDMOAD syndrome. J. Pediatr. 114: 405-410.

4. Swift, R.G., D.B. Sadler, and M. Swift. 1990. Psychiatric findings in Wolfram syndrome homozygotes. Lancet. 336:667-669.

5. Swift, R.G., D.O. Perkins, C.L. Chase, D.B. Sadler, and M. Swift. 1991 Psychiatric disorders in 36 families with Wolfram syndrome. Am. J. Psychiatry. 148:775-779.

6. Cremers, C.W., P.G. Wijdeveld, and A.J. Pinckers. 1977. Juvenile diabetes mellitus, optic atrophy, hearing loss, diabetes insipidus, atonia of the urinary tract and bladder, and other abnormalities (Wolfram syndrome): a review of 88 cases from the literature with personal observations on three new patients. Acta Paediatr. Scand. 264:1-16.

7. Fraser, G.C., and T. Gunn. 1977. Diabetes mellitus, diabetes insipidus and optic atrophy. An autosomal recessive syndrome? J. Med. Genet. 14:190193.

8. Polymeropoulos, M.H., R.G. Swift, and M. Swift. 1994. Linkage of the gene for Wolfram syndrome to markers on the short arm of chromosome 4 Nat. Genet. 8:95-97.

9. Bundey, S., K. Poulton, H. Whitwell, E. Curtis, I.A.R. Brown, and A.R. Fielder. 1992. Mitochondrial abnormalities in the DIDMOAD syndrome. J. Inherited Metab. Dis. 15:315-319.

10. Rötig, A., V. Cormier, P. Chatelain, R. Francois, J. Saudubray, P. Rustin, and A. Munnich. 1993. Deletion of mitochondrial DNA in a case of earlyonset diabetes mellitus, optic atrophy, and deafness (Wolfram syndrome, MIM 222300). J. Clin. Invest. 91:1095-1098.

11. Giles, R.E., H. Blanc, R.M. Cann, and D.C. Wallace. 1980. Maternal inheritance of human mitochondrial DNA. Proc. Natl. Acad. Sci. USA. 83:96119615 .

12. Zeviani, M., N. Bresolin, C. Gellera, A. Bordoni, M. Pannaci, P. Amati, M. Moggio, S. Servidei, F. Scarlato, and S. DiDonato. 1990. Nucleus-driven large-scale deletions of the human mitochondrial genome: a new autosomal dominant disease. Am. J. Hum. Genet. 47:904-914.

13. Yuzaki, M., N. Ohkoshi, I. Kanazawa, Y. Kagawa, and S. Ohta. 1989. Multiple deletions in mitochondrial DNA at direct repeats of non-D-loop region in cases of familial mitochondrial myopathy. Biochem. Biophys. Res. Commun. 164:1352-1357.

14. Uncini, A., S. Servidei, G. Silvestri, G. Manfredi, M. Sabateli, A. Di Muzio, E. Richhi, M. Mirabella, S. Di Mauro, and P. Tonali. 1994. Ophthalmoplegia, demyelinating neuropathy, leukoencephalopathy, myopathy, and gastrointestinal dysfunction with multiple deletions of mitochondrial DNA: a mitochondrial multisystem disorder in search of a name. Muscle \& Nerve. 17: 617-674.

15. Suomalainen, A., J. Kaukonen, P. Amati, R. Timonen, M. Haltia, J. Weissenbach, M. Zeviani, H. Somer, and L. Peltonen. 1995. An autosomal locus predisposing to deletions of mitochondrial DNA. Nat. Genet. 9:146-151.

16. Rustin, P., D. Chretien, T. Bourgeron, B. Gerard, A. Rötig, J.M. Saudubray, and A. Munnich. 1994. Biochemical and molecular investigations in respiratory chain deficiencies. Clin. Chim. Acta. 228:35-51.

17. Goto, Y., I. Nonaka, and S. Horai. 1990. A mutation in the tRNA(Leu)(UUR) gene associated with the MELAS subgroup of mitochondrial encephalomyopathies. Nature (Lond.). 348:651-653.

18. DiDonato, S., M. Zeviani, P. Giovannini, N. Savarese, M. Rimoldi, C. Mariotti, F. Girotti, and T. Caraceni. 1993. Respiratory chain and mitochondrial DNA in muscle and brain in Parkinson's disease patients. Neurology. 43: 2262-2268.

19. Ott, J. 1991. Analysis of Human Genetic Linkage. Johns Hopkins University Press, Baltimore, MD. p. 79.

20. Cottingham, R.W., R.M. Idury, and A.A. Schäffer. 1993. Faster sequential genetic linkage computations. Am. J. Hum. Genet. 53:252-263.

21. Gyapay, G., J. Morissette, A. Vignal, C. Dib, C. Fizames, Ph. Millasseau, S. Marc, G. Bernardi, M. Lathrop, and J. Weissenbach. 1994. The 1993-94 Généthon human genetic linkage map. Nat. Genet. 7:246-339.

22. Ott, J. 1989. Computer-simulation methods in human linkage analysis. Proc. Natl. Acad. Sci. USA. 86:4175-4178.

23. Weeks, D.E., J. Ott, and G.M. Lathrop. 1990. SLINK: a general simulation program for linkage analysis. Am. J. Hum. Genet. 47(Suppl.):A204.

24. Casademont, J., A. Barrientos, F. Cardellach, A. Rötig, J.M. Grau, J. Montoya, B. Beltrán, F. Cervantes, C. Rozman, X. Estivill, et al. 1994. Multiple deletions of mtDNA in two brothers with sideroblastic anaemia and mitochondrial myopathy and in their asymptomatic mother. Hum. Mol. Genet. 3:19451949.

25. Cormier, V., A. Rötig, M. Tardieu, M. Colonna, J.M. Saudubray, and A. Munnich. 1991. Autosomal dominant deletions of the mitochondrial genome in a case of progressive encephalomyopathy. Am. J. Hum. Genet. 48:643-648.

26. Cortopassi, G.A., and N. Arnheim. 1990. Detection of a specific mitochondrial DNA deletion in tissues of older human. Nucleic Acids Res. 18:69276933.

27. Corral-Debrinski, M., T. Horton, M.T. Lott, J.M. Shoffner, M. Flint Beal, and D.C. Wallace. 1992. Mitochondrial DNA in human brain: regional variability and increase with advanced age. Nat. Genet. 2:324-329. 
28. Polymeropoulos, M.H., H. Xiao, E.F. Torrey, L.E. Delisi, T. Crow, and C.R. Merril. 1992. Search for a genetic event in monozygotic twins discordant for schizophrenia. Psychiatry Res. 48:27-36.

29. Hsieh, C.L., T.A. Donlon, B.T. Darras, D.D. Chang, J.N. Topper, D.A. Clayton, and U. Francke. 1990. The gene for the RNA component of the mitochondrial RNA-processing RNA endoribonuclease is located on human chromosome 9p and on mouse chromosome 4. Genomics. 6:540-544.

30. Tiranti, V., E. Rossi, A. Ruíz-Carrillo, G. Rossi, M. Rocchi, S. DiDo- nato, O. Zuffardi, and M. Zeviani. 1995. Chromosomal localization of mitochondrial transcription factor A (TCF6), single-stranded DNA binding protein (SSBP) and endonuclease G (ENDOG), three human housekeeping genes involved in mitochondrial biogenesis. Genomics. 25:559-564

31. Anderson, S., A.T. Bankier, B.G. Barrell, M.H.L. Bruijn, A.R. Coulson, J. Drouin, I.C. Eperon, D.P. Nierlich, B.A. Roe, F. Sanger, et al. 1981. Sequence and organization of the human mitochondrial genome. Nature (Lond.). 290:457-465. 